\title{
Active control does not eliminate motion-induced illusory displacement.
}

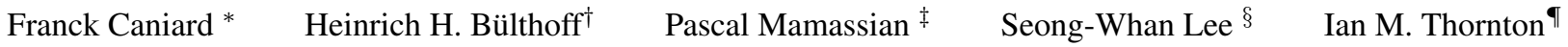

\begin{abstract}
When the sine-wave grating of a Gabor patch drifts to the left or right, the perceived position of the entire object is shifted in the direction of local motion. In the current paper, we explored whether active control of the physical position of the patch can overcome such motion induced illusory displacement. We created a simple computer game and asked participants to continuously guide a $\mathrm{Ga}-$ bor patch along a randomly curving path. When the grating inside the Gabor patch was stationary, participants could perform this task without error. When the grating drifted to either left or right, we observed systematic errors consistent with previous reports of motion-induced illusory displacement. Specifically, when the grating drifted to the right, participants adjusted the global position of the patch to the left of the target line, and when it drifted to the left, errors were to the right of the line. The magnitude of the errors was consistent with previously reported perceptual judgements for centrally presented items, and scaled systematically with the speed of local drift. Importantly, we found no evidence that participants could adapt or compensate for illusory displacement given active control of the target. The current findings could have important implications for interface design, suggesting that local dynamic components of a display could affect perception and action within the more global application environment.
\end{abstract}

CR Categories: J.4 [Social and Behavioral Sciences]: Psychology H.1.2 [Models and Principles]: User/Machine Systems-Human information processing H.5.2 [Information interfaces and Presentation]: User Interfaces-Input Devices and Strategies

Keywords: perception and action, localisation, local motion, global motion, illusions, Gabor, joystick control, adaptation, tracking

Links: $\odot$ DL 国PDF

\footnotetext{
*Max Planck Institute for Biological Cybernetics, Tübingen, Germany. E-Mail: franck.caniard@ tuebingen.mpg.de

${ }^{\dagger}$ Max Planck Institute for Biological Cybernetics and Department of Brain and Cognitive Engineering, Korea University. E-Mail: heinrich.buelthoff@tuebingen.mpg.de)

${ }^{\ddagger}$ Laboratoire Psychologie de la Perception, Université Paris Descartes, Paris, France. E-Mail: pascal.mamassian@parisdescartes.fr

$\S$ Department of Brain and Cognitive Engineering, Korea University, Seoul, South Korea. E-Mail: swlee@image.korea.ac.kr

ฯ Psychology Department, Swansea University, Swansea, UK. E-Mail: i.m.thornton@swansea.ac.uk)
}

Copyright $(2011$ by the Association for Computing Machinery, Inc.

Permission to make digital or hard copies of part or all of this work for personal or classroom use is granted without fee provided that copies are not made or distributed for commercial advantage and that copies bear this notice and the full citation on the first page. Copyrights for components of this work owned by others than ACM must be honored. Abstracting with credit is permitted. To copy otherwise, to republish, to post on servers, or to redistribute to lists, requires prior specific permission and/or a fee. Request permissions from Permissions Dept, ACM Inc., fax +1 (212) 869-0481 or e-mail permissions@acm.org.

APGV 2011, Toulouse, France, August 27 - 28, 2011

(C) 2011 ACM 978-1-4503-0889-2/11/0008 $\$ 10.00$

\section{Introduction}

Under laboratory conditions, it has often been shown that motion can influence the perceived position of static objects. This is true, for example, when the test item used to probe a motion aftereffect (MAE) apparently shifts in the direction opposite to adapted motion [Nishida and Johnston 1999; Snowden 1998]. Similarly, it has been shown that peripheral motion can capture briefly presented central targets, shifting their perceived position in the direction of motion [Whitney and Cavanagh 2000; Whitney and Cavanagh 2002]. Of particular interest in the current paper are situations in which local motion within an object also influences the perception of global position [Ramachandran and Anstis 1990; De Valois and De Valois 1991].

One common version of this motion-induced illusory displacement is illustrated in Figure 1. When the sine-wave grating component of a Gabor patch is made to drift locally either to the left or right, the global position of the entire patch appears shifted in that direction, even though the physical position of the patch does not change [De Valois and De Valois 1991; Fu et al. 2004]. Although the magnitude of this Gabor-based motion-induced illusory displacement is typically small - ranging between 2 and 15 min arc for centrally presented targets [De Valois and De Valois 1991; Kerzel et al. 2008; Tsui et al. 2007] - it is highly robust and has proven to be a very useful experimental tool for exploring the relationship between motion and position [Bressler and Whitney 2006; Chung et al. 2007; Fu et al. 2004; Kerzel and Gegenfurtner 2005; Rider et al. 2009; Yamagishi et al. 2001].

Several low-level explanations have been proposed to account for such errors, including active extrapolation [De Valois and De Valois 1991], changes to receptive field properties [Fu et al. 2004], changes to the perceived size and shape of the patch [Tsui et al. 2007] and changes to the perceived contrast of target boundaries [Arnold et al. 2007; Whitney et al. 2003]. Recent evidence also suggests that at least some forms of motion-dependent illusory displacement occur relatively late in visual processing, certainly after the stage of motion integration [Hisakata and Murakami 2009; Mather and Pavan 2009; Rider et al. 2009]. In general, it is felt that understanding such illusory displacement has the potential to shed light on how the visual system constructs and maintains a stable representation of the world (see [Whitney 2002; Khurana and Nijhawan 2010] for more detailed discussions).

One issue that continues to puzzle researchers, not only in relation to the above motion-induced displacement errors, but also with respect to other forms of visual mislocalisation for example, the Fröhlich Effect [Müsseler and Aschersleben 1998], the Onset Repulsion Effect [Thornton 2002], Representational Momentum [Freyd and Finke 1984] and the Flash Lag Effect [Nijhawan 1994] is why our behaviour outside of the laboratory is not more error prone. That is, given the existence of illusory displacement, how are we able to function so effectively during our interactions with the many moving and static objects that populate our day-today environments? How are we able to cross a busy street, catch a falling child or intercept a flying Frisbee? Possibly the magnitude of the errors we measure in the laboratory are simply too small? Or perhaps they rely on unnaturally sparse visual environments? A more interesting possibility, and the one we investigate here, is that the involvement of action-related systems compensates for errors arising during perception. 

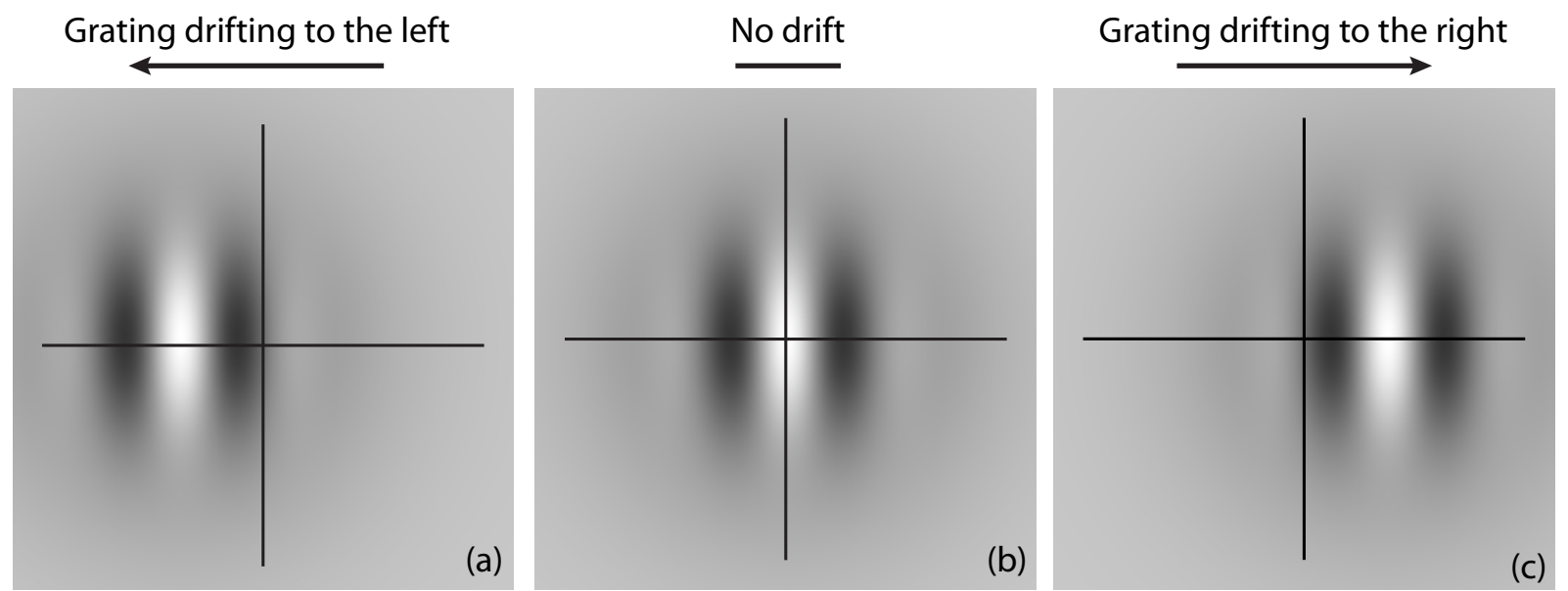

Figure 1: The effect of motion-induced illusory displacement on perceived global position. In all panels, the crosshair indicates the true global position of the Gabor patch. When the grating within the patch drifts to the left (panel a) or right (panel c), perceived global position is also shifted in that direction. In the absence of local motion (panel b), perceived and physical position align.

The precise relationship between perception and action continues to be a topic of much debate. Functionally, there is no question that these two aspects of behaviour are tightly linked. The nature of that link, or more specifically, the representations that might support it, continues to be controversial. At one extreme are those who propose that perception and action share a common representational framework [Hommel et al. 2001; Prinz 1997]. At the other, are those who have argued that conscious perception and vision-for-action are served by two separate representational systems [Goodale and Milner 1992; Goodale and Westwood 2004; Milner and Goodale 1995]. An important, though highly disputed, source of evidence in support of the latter position have been claims that actions are to some degree immune for visual illusions [Aglioti et al. 1995] but see [Bruno 2001; Carey 2001; Franz 2001; Franz et al. 2003; Franz et al. 2001; Franz and Gegenfurtner 2008; Glover 2004; Smeets and Brenner 2001].

Two previous studies have specifically addressed whether the impact of motion- induced illusory displacement varies depending on whether perceptual or action- based responses are required. [Yamagishi et al. 2001] found that pointing to the remembered location of a drifting Gabor patch actually led to larger errors than judging its position using a visible ruler. [Kerzel and Gegenfurtner 2005] compared pointing to a range of perceptual judgements. They found that, depending on the precise nature of the perceptual judgement employed, action- based errors could be equal, larger or smaller. As these authors note "The answer to the question of which measure shows the largest error depends strongly on the specific methods. The more general point is that the comparison between perception and action is elusive because of the fundamental problem of choosing an appropriate comparison (probe) stimulus in the perceptual task" ([Kerzel and Gegenfurtner 2005]; p200; see also [Franz and Gegenfurtner 2008]).

In the current paper, we have tried to avoid this "fundamental problem" by taking a different approach. Rather than comparing perception and action in separate conditions, we measured them together, while at the same time trying to maximize the role of action. To do this we created a very simply computer game that gave participants continuous, active control of the physical position of a Gabor patch at all times. Using a joystick, we asked them to steer the patch along a randomly curving path keeping it centred on the line. The vertical position of the patch was fixed, but participants could control horizontal (left/ right) movement. The speed and direction of the grating drift was varied and we measured the accuracy with which they could keep the patch on the curve, sampling at $40 \mathrm{~Hz}$. If action accounts for error free performance in the face of illusory displacement, then we would expect participants to quickly adapt to its presence in the current task and for errors to be negligible or absent. Were illusory displacement to continue to affect performance, movement errors should be in the direction opposite to local drift, signalling attempts to compensate for perceived shifts in global position.

In addition to the basic research goals associated with motioninduced illusory displacement, the current study also has relevance in several applied domains. For example, in the context of both heads-up technology and more standard navigation and control display panels, dynamic information delivery in many types of vehicle is now common. Understanding whether local motion within such displays has an impact on the global control of the vehicle or even the efficiency of actions directed at the interfaces themselves (e.g. touch-screen interactions) has both design and potentially even safety implications.

\section{Methods}

\subsection{Participants}

Seven members of the Max Planck Institute for Biological Cybernetics Psychophysics laboratory took part in this experiment on a voluntary basis. All observers had normal or corrected to normal vision. Observers were not informed about the specific goals of the research until after the final experimental session ended.

\subsection{Equipment}

Stimuli were displayed and data collected on a Macintosh G4 computer connected to a standard LCD display. A USB Joystick was placed centrally on the desk directly in front of the screen and served as the only input device. The viewing distance was approximately $70 \mathrm{~cm}$ and the participants were free to move their head and eyes at all times. Custom written software was used in all aspects of stimulus presentation and data collection. 


\subsection{Stimuli \& Display}

Figure 2 illustrates the display used in the current study, along with the basic experimental set-up. A moving path was drawn in black at the centre of a middle-grey display area and animated to give the impression of forward motion by scrolling downwards from top to bottom at a constant speed of $2.7 \mathrm{DVA} /$ second. The path was constructed by assembling pre-defined square tiles in a random order. One period of a sine wave was drawn vertically on each tile, from the bottom centre to the top centre, so that any 2 tiles assembled seamlessly. The curves could deviate to left or right of the centre and had peak amplitudes of $0.7,1.4$ or $2.1^{\circ}$ visual angle and a fixed vertical height of $4.2^{\circ}$. The shape of the path was constrained by randomly choosing different amplitudes to give non-repeating sequences of curves.

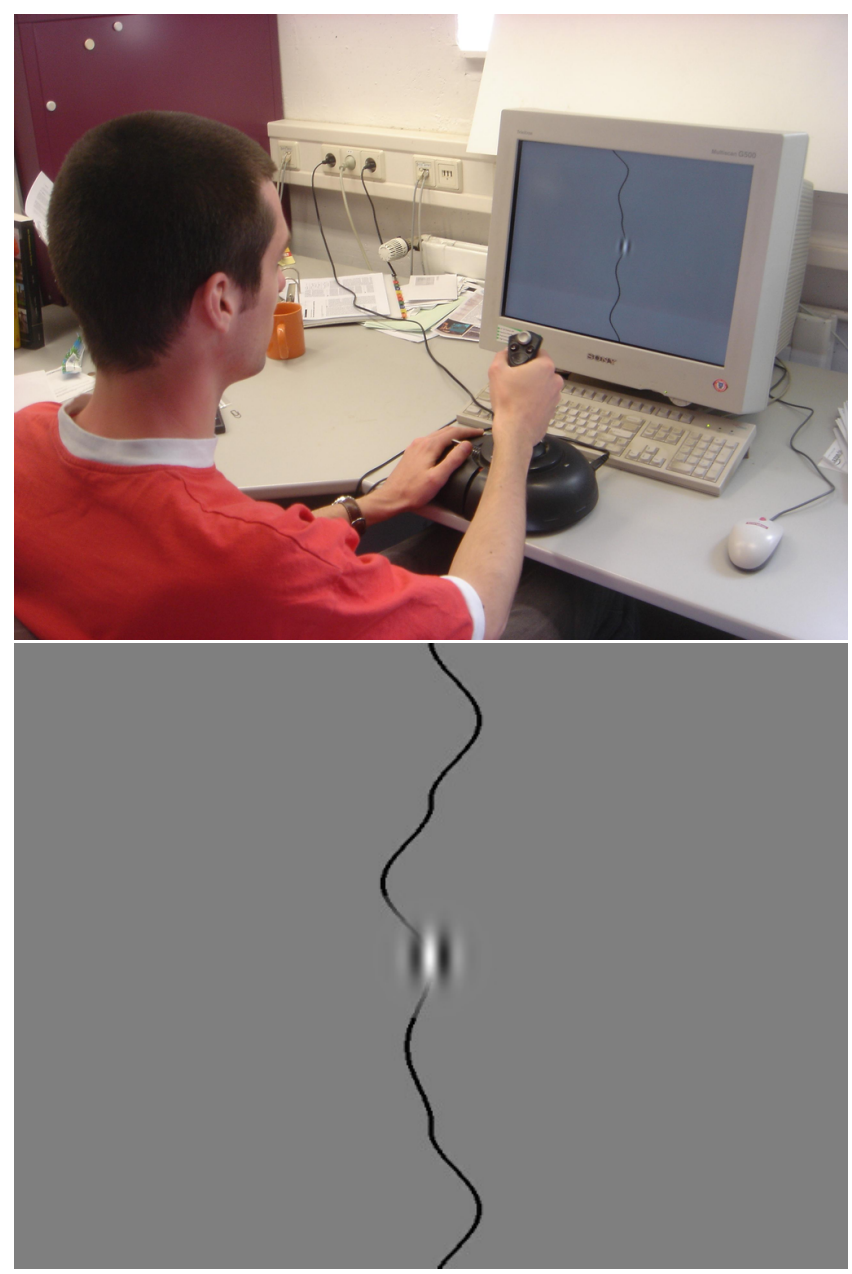

Figure 2: Experimental setup and stimulus display. See text for details.

The target object was a low-frequency Gabor patch. That is, a vertical sine wave grating with a spatial frequency of $1 \mathrm{cycle} /{ }^{\circ}$ and $80 \%$ contrast that was windowed by a Gaussian envelope $\left(\mathrm{SD}=0.5^{\circ}\right)$, giving a visible spatial extent of approximately $2.4^{\circ}$. The grating within the Gabor patch was either stationary or drifted to the left or right. In each session, the patch was initially stationary for 10 seconds and would then begin to drift either left or right at a fixed rate. The direction of initial drift was random and continued for 30 seconds before switching to the opposite direction. Each session consisted of 8 left/right switches, thus lasting approximately 5 min- utes. The speed of drift was varied in separate sessions. The drift speeds were $0,0.5,1.0,1.5,2.0,2.5$ or $3.0^{\circ} / \mathrm{sec}$. The order of presentation of these speed manipulations was randomised separately for each observer.

\subsection{Task \& Measure}

The observers' task was to use the joystick to guide the target patch along the pathway as accurately as possible. More specifically, the goal was to keep the patch centred on the pathway while following the curves without deviation. The vertical position of the target patch was fixed in the centre of the screen, with the only degree of freedom being the left-right horizontal position. The horizontal position of the patch was continuously monitored at $40 \mathrm{~Hz}$, providing very precise measurement of whether the physical centre of the patch was on the line, or was shifted to the left or right. The distance between the patch centre and the curve thus gave rise to our dependent measure, which we will call Control Error. Note that, illusory displacement caused by local drift to the right, should give rise to over-compensating movements to the left, and vice versa. In all results and figures reported below, positive errors refer to the situation where the patch was physically positioned to the right of the curve, and negative errors refer to where the patch was physically positioned to the left.

An analogue joystick was used so that we could measure not only the direction of inclination of the stick, but also how far away from the central position it was pushed. The experimental software used that information to adjust the translation speed of the Gabor patch. This made it possible to smoothly follow all possible curvatures of the path, as well as perform very small adjustments of the patchs position.

\subsection{Procedure}

Participants were first made familiar with the general nature of the display, the task and the method by completing a brief demonstration session with a stationary Gabor patch. After this initial training, each participant completed 7 separate 5-minute sessions in which the grating within the patch drifted. The speed was constant within sessions, but different for each individual session. The order of speed/session was randomized separately for each participant. During each session, the patch was initially stationary for $10 \mathrm{sec}-$ onds to give participants time to settle before data collection began. After that, the patch started to drift in a randomly chosen initial direction, which alternated every 30 seconds. Each session contained 8 blocks of 30 seconds ( 4 in each direction). The initial direction of drift was randomized and direction alternated in each successive 30 second. Participants were encouraged to take short breaks between blocks and the entire experiment lasted approximately 45 minutes.

\subsection{Design \& Data Analysis}

Data were analysed using a 2 (Local Drift: Left or Right) x 2 (Global Motion - Physical Movement: Left or Right) x 7 (Grating Speed: $0,0.5,1.0,1.5,2.0,2.5$ or $\left.3.0^{\circ} / \mathrm{s}\right)$ x 4 (30 second block) repeated measures Analysis of Variance. The Global Motion factor reflects the fact that in contrast to most previous studies of illusory displacement, in the current study the physical position of the patch could really be changing from left to right in response to joystick movements, in addition to Local Drift within the patch. To explicitly measure the impact of Global motion we measured at each time point whether the current position resulted from a left or right movement of the joystick. 


\section{Results}

Figure 3 illustrates the impact that local motion had on the active control of the target patch. It is clear that for all observers, local motion in one direction led to control errors in the opposite direction, consistent with a reaction to illusory displacement. Although there was some variation in the magnitude of the control errors, all seven participants showed the same basic pattern. When the grating drifted to the left, the patch was placed to the right of the control line $(\mathrm{M}=12.1 \mathrm{~min}$ arc $)$ and when it drifted to the right, control errors were to the left $(\mathrm{M}=-11.4 \mathrm{~min}$ arc $)$. This pattern, consistent with attempts to compensate for motion induced illusory displacement, led to a reliable main effect of local motion $\mathrm{F}(1,6)=68.8$, $\mathrm{MSE}=1584, \mathrm{p}<0.001$.

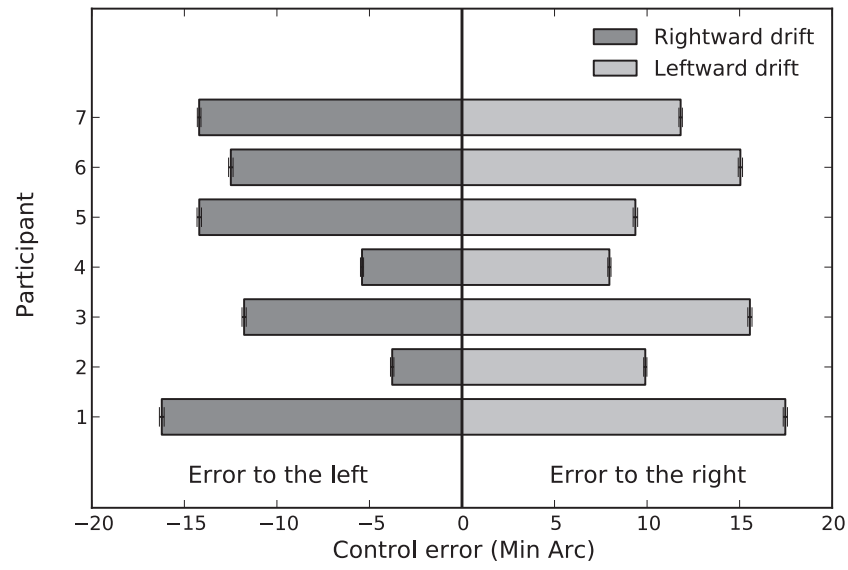

Figure 3: Control error per participant as a function of local drift direction, averaged over speed and block. For all participants, drift in one direction leads to consistent over-correction in the opposite direction. In this and all other figures, negative values indicate positional errors to the left of the target curve and positive values, errors to the right. Error bars illustrate the standard error of the mean.

Figure 4 shows how the speed of local motion drift affected performance. When the patch was stationary, there was essentially no direction error, indicating that the basic tracking task was possible to perform. Immediately after the patch began to drift, however, directional errors appear, rising from an initial level of approximately $8 \mathrm{~min}$ arc at $0.5^{\circ} / \mathrm{s}$ to a maximum of just over $15 \mathrm{~min}$ arc at $3^{\circ} / \mathrm{s}$. For both left and rightward drift, there is an initial period of exponential increase with speed, which appears to reach asymptote slightly earlier for rightward drift $\left(1.5^{\circ} / s\right)$ than for leftward drift $\left(2^{\circ} / s\right)$. For both directions, a third order polynomial accounted for more than $99 \%$ of the variance. To better capture the general influence of speed, we analysed the absolute magnitude of errors, rather than including the sign of the direction shift. This revealed a clear main effect, $\mathrm{F}(6,36)=6.14, \mathrm{MSE}=262, \mathrm{p}<.001$, and no interaction between speed and direction.

Figures 5 and 6 summarise how control error varied across time. As can be seen in Figure 5, there was no indication that errors either increased or decreased as participants were repeatedly exposed to drift in the four direction blocks that made up each of the speed sessions. Consistent with this pattern, there was no main effect of block, nor any interactions involving block. Separate analysis of joystick precision did indicate a slight trend for less variability in later blocks, although this trend did not reach significance, $\mathrm{F}(3,18)$ $=2.78, \mathrm{MSE}=0.001, \mathrm{p}=.09$. To examine behaviour within the 30 -second epochs that made up each block, we can also plot the

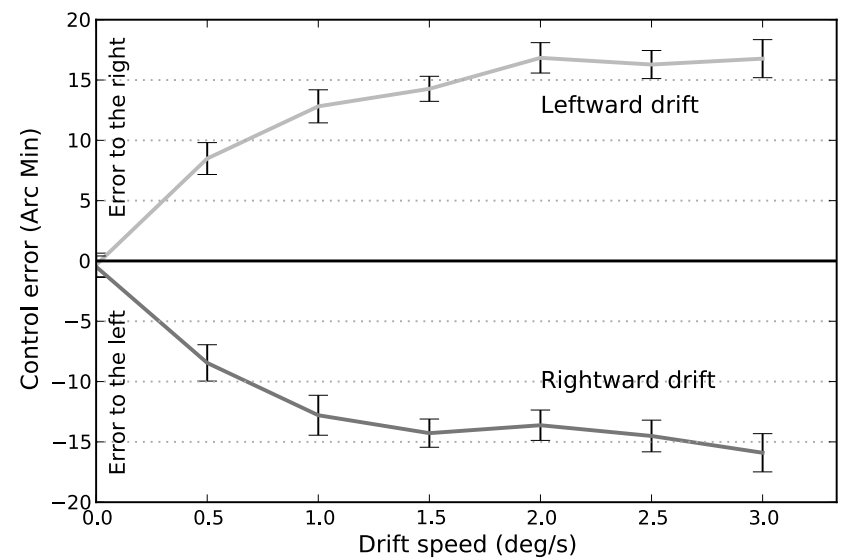

Figure 4: Control error as a function of the speed of local drift.

average error as a function of time-on-task. This is shown in Figure 6. Again, there appears to be no systematic change in the slope of this function away from zero, which would indicate a change in response to the illusory displacement as a function of time. As the functions shown in Figure 6 are collapsed across all speeds and repetitions of the 30 second epochs for all participants, the initial instability below about 3 seconds would appear to reflect the adjustment to the change in Gabor drift from right-to-left or vice versa.

The only other significant finding was a main effect of Global Motion, $\mathrm{F}(1,6)=13.4$, MSE $=1747, \mathrm{p}<0.05$. Across all conditions errors were slightly further to left when the joystick was physically moving the patch to the left $(M=-5.1)$, relative to when the patch was being moved to the right $(\mathrm{M}=5.8)$. As Global motion did not interact with either Speed or Local Drift, it seems most likely that this main effect reflects some aspect of joystick motor control, rather than a direct modulation of motion induced illusory displacement.

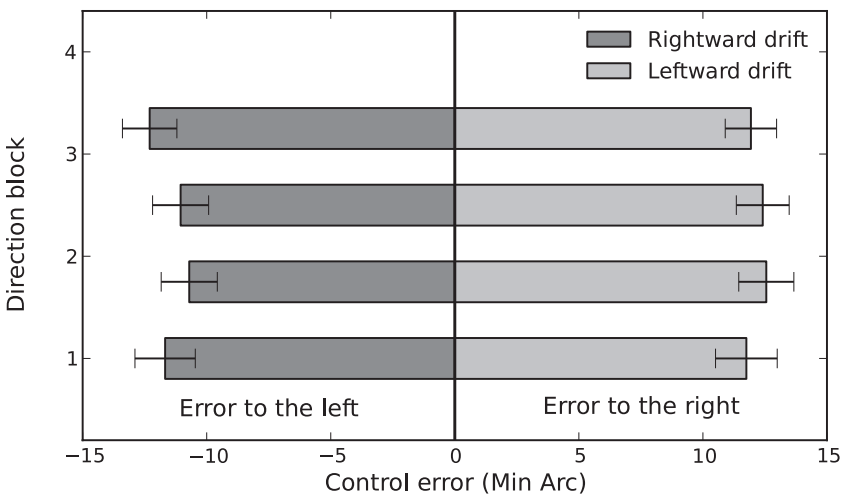

Figure 5: Change in control error as a function of block.

\section{Discussion}

To explore the impact of active control on motion-induced illusory displacement, we created a simple computer game and asked participants to continuously guide a Gabor patch along a randomly curving path. When the grating inside the Gabor patch was stationary, participants could perform the task without error. When the grating drifted to either left or right, we observed systematic errors consistent with previous reports of motion-induced illusory displacement. Specifically, when the grating drifted to the right, participants ad- 


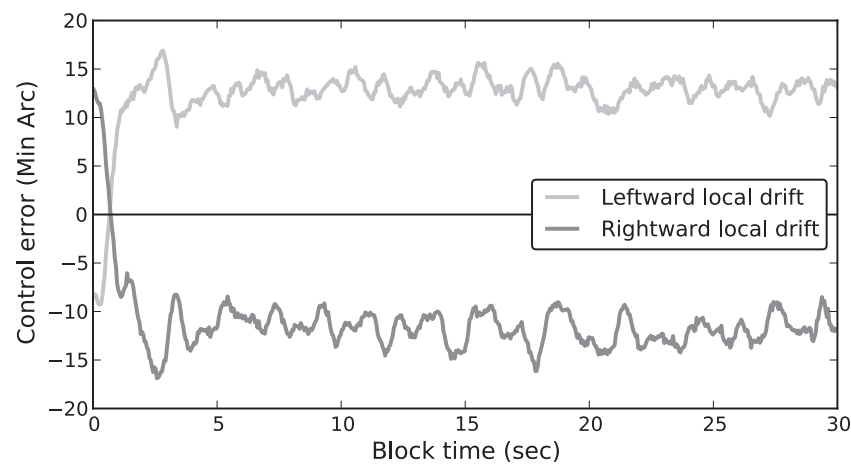

Figure 6: Change in Control error within a 30 second epoch.

justed the global position of the patch to the left of the target line. This suggests that in order for them to perceive the patch as centred, they had to continually correct for illusory displacement and physically move the patch against the flow of the local drift. Similarly, when the grating drifted to the left, errors were to the right of the line. It thus appears that perception of the global position of the patch is continuously affected by local drift, and that active control cannot eliminate the tracking errors that subsequently arise.

We believe that in providing continuous, active control of the target patch, and in sampling participant errors at $40 \mathrm{~Hz}$, we have created a sensitive assessment of the impact of action on this form of illusory displacement. Clearly, there is no evidence that such active control leads to error-free performance, as we had speculated in the Introduction. On the contrary, the magnitude of the errors found in the current study would appear to be on the higher end of the 2-15 min arc range previously estimated for perception alone [De Valois and De Valois 1991; Kerzel et al. 2008; Tsui et al. 2007]. Indeed, those estimates also include peripherally presented targets, and it is known that illusory displacement scales with eccentricity, increasing by 1-2 min arc per degree [Chung et al. 2007; De Valois and De Valois 1991; Fu et al. 2004]. Thus, our current results, using a centrally presented target, may be a conservative estimate of the impact of illusory displacement. It would be interesting to adapt our task to explore active tracking in the periphery.

Another well-documented characteristic of illusory displacement errors is their temporal frequency response profile. With the relatively low range of velocities considered in the current paper, $(0$ $3^{\circ} / \mathrm{s}$ ) the gradual increase and then asymptote of errors shown in Figure 4, nicely mirrors the data obtained in previous studies with perceptual judgements (e.g., [Bressler and Whitney 2006], Figure 7b; [Chung et al. 2007], Figure 3; [De Valois and De Valois 1991], Figure 3). At much higher velocities (e.g., $>10^{\circ} / s$ ), the size of illusory displacement tends to decrease and even disappear [Bressler and Whitney 2006]. Several authors [De Valois and De Valois 1991; Bressler and Whitney 2006] have noted how this band bass tuning is similar to the well-known temporal contrast sensitivity function [Kelly 1979], but quite distinct from motion-sensitivity profiles, which are essentially low-pass [Nakayama 1985]. It would be interesting to see if a similar decay at high velocities also occurs with the current action-related task.

Despite the similarity in both the magnitude and overall pattern of errors between the current data and previous research, the lack of an explicit perceptual control condition is a limiting factor. That is, we have no way of establishing whether the current display would give rise to larger, smaller or equal perceptual judgements. As noted in the Introduction, our avoidance of such a control condition was partly motivated by the notorious general problem of appropriately matching perception and action tasks in the context of illusions.
As [Gegenfurtner and Franz 2007] note "A safe conclusion from these numerous studies is that comparing the effects of visual illusions for perception and action is difficult" (p2; see also [Franz and Gegenfurtner 2008] for some detailed pitfalls). The continuous nature of the current task, and our ability to sample action at $40 \mathrm{~Hz}$, makes the issue of appropriate perceptual control even more extreme.

In order to make our task more amenable for perception/action comparisons as well as to provide the opportunity to collect data from much larger sample sizes than is typical in psychophysical studies - we are currently developing an iPad version of our task. This involves using the accelerometer-based tilt control of that device to guide the patch through discrete "gates". The analogous real-world tasks might include a slalom ski course, guiding a boat through channel markers or taking a horse around a show jumping course. The addition of "gates" allows us to keep the continuous nature of the task, but to also have discrete events with which we can probe perception. Our goal is to compare the action trajectory through the gates with the ability to accurately judge whether the patch passes to the left or right of centre. In the perceptual task, we will parametrically vary the physical offset of the patch as it passes through the gate and use psychophysical methods to characterize the level of performance (i.e. sensitivity and bias). With these modifications, we hope to be able to more specifically quantify the relationship between perception and action in this task.

One particularly interesting aspect of the current data was that we could find no evidence of adaption to illusory displacement over time. That is, there was no apparent increase or decrease in error direction or magnitude across the four block repetitions that made up each speed session. There was a slight hint that joystick control became more precise in later blocks, suggesting that practice had some impact, but this marginal learning effect appears to be unrelated to displacement error. Examining the time course within the 30 second blocks also revealed no change. As speed was counterbalanced across participants, our design does not allow us to examine session-to-session changes (i.e., the effects of speed and time would be confounded), but our strong intuition is that no changes would be apparent at this time resolution either.

Rapid adaptation to both spatial and temporal offsets, are known to occur at various levels within the nervous system [Bedford 1993; Cunningham et al. 2001b; Cunningham et al. 2001a; Fajen 2007; Miall and Jackson 2006; Welch 1978]. Low-level adaptive responses to the local motion within the patch, or higher-level recalibration based on detecting and trying to correct for the illusory displacement would both have predicted a reduction in errors. The alternating, relatively short periods of constant local motion might account for the lack of low-level, direction-selective fatigue effects. Similarly the absence of explicit feedback may have prevented recalibration. That is, the nature of the illusory displacement is such that participants have no perceptual information available to inform them that they are deviating to the left or right of the curve. Without additional feedback of some kind, they may simply be unable to adapt. If this explanation is correct, and if the action system were unaffected by illusory displacement, as we speculated in the Introduction, then clearly, error signals originating from that source do not appear to be propagating to perception, or at least are not being used to re-calibrate overall performance.

Finally, we note that the current findings have potentially important implications for interface and display design. Specifically, if information delivery involves local dynamic components, such as scrolling text, explicit direction indicators or animated icons, then these components could influence perception of position either within the local reference frame of the display device itself, or more globally. Furthermore, as we have seen here, apparent changes in 
perceived position also have systematic and robust effects on related actions. Heads-up or augmented reality displays, in which local information dynamics and global perception of position are intentionally overlapped, could be particularly prone to the sorts of errors we have measured here. Control and navigation systems that employ motion, particularly those requiring rapid, touch-screen responses, may also prove to be susceptible to motion-induced illusory displacement. Clearly, additional research specifically targeting these types of application domains would seem appropriate.

In conclusion, we have designed a task that we believe makes a fair assessment of the impact of motion-induced illusory displacement on active control. Both the magnitude and the pattern of the errors we observed suggest that action systems are unable to adapt or compensate for the presence of this form of visual illusion. We thus find no support for the notion that the involvement of action per se accounts for error free performance in our day-to- day encounters with dynamic objects.

\section{Acknowledgements}

The authors would like to thank Andries Hof and Jo Messa for help with data collection, George Mather, Astros Chatziastros and Bettina Friedrich for useful discussions. This research was supported by WCU (World Class University) program funded by the Ministry of Education, Science and Technology through the National Research Foundation of Korea (R31-10008).

\section{References}

Aglioti, S., DeSouza, J., And Goodale, M. A. 1995. Sizecontrast illusions deceive the eye but not the hand. Current Biology 5, 679-685.

Arnold, D. H., Thompson, M., And Johnston, A. 2007. Motion and position coding. Vision Research 47, 2403-2410.

Bedford, F. L. 1993. Perceptual learning. Psychology of Learning and Motivation. Academic Press.

Bressler, D. W., AND Whitney, D. 2006. Second-order motion shifts perceived position. Vision Research 46, 1120-1128.

BRUnO, N. 2001. When does action resist visual illusions? Trends in Cognitive Sciences 5, 379-382.

CAREY, D. P. 2001. Do action systems resist visual illusions? Trends in Cognitive Sciences 5, 109-113.

Chung, S. T. L., Patel, S. S., Bedell, H. E., and Yilmaz, O. 2007. Spatial and temporal properties of the illusory motioninduced position shift for drifting stimuli. Vision Research 47, 231-243.

Cunningham, D. W., Billock, V. A., And Tsou, B. H. 2001. Sensorimotor adaptation to violations of temporal contiguity. Psychological Science 12, 532-535.

Cunningham, D. W., Chatziastros, A., von der Heyde, M., AND BÜLthofF, H. 2001. Driving in the future: Temporal visuomotor adaptation and generalization. Journal of Vision $1(2), 88: 98$.

De Valois, R., And De Valois, K. 1991. Vernier Acuity With Stationary Moving Gabors. Vision Res. 31, 9, 1619-1626.

FAJEN, B. R. 2007. Rapid recalibration based on optic flow in visually guided action. Experimental Brain Research 183, 6174.
Franz, V. H., And Gegenfurtner, K. R. 2008. Grasping visual illusions: Consistent data and no dissociation. Cognitive Neuropsychology 25, 920-950.

Franz, V. H., Fahle, M., BÜlthoff, H., and GegenfurtNER, K. 2001. Effects of visual illusions on grasping. Journal of Experimental Psychology - Human Perception and Performance $27,1124-1144$.

Franz, V., Bülthoff, H., And Fahle, M. 2003. Grasp effects of the Ebbinghaus illusion: Obstacle-avoidance is not the explanation. Experimental Brain Research 149(4), 470-477.

FRANZ, V. 2001. Action does not resist visual illusions. Trends in Cognitive Sciences 5, 457-459.

FREYD, J. J., AND FINKE, R. A. 1984. Representational momentum. Journal of Experimental Psychology: Learning, Memory, and Cognition 10, 126-132.

Fu, Y.-X., Shen, Y., GaO, H., AND Dan, Y. 2004. Asymmetry in visual cortical circuits underlying motion-induced perceptual mislocalization. Journal of Neuroscience 24, 2165-2171.

Gegenfurtner, K. R., And Franz, V. H. 2007. A comparison of localization judgments and pointing precision. Journal of Vision 7(5):11, 1-12.

GLOVER, S. 2004. Separate visual representations in the planning and control of action. Behavioral and Brain Sciences 27, 3-23.

Goodale, M. A., And Milner, A. D. 1992. Separate visual pathways for perception and action. Trends in Neuroscience 15, $20-25$.

Goodale, M. A., And Westwood, D. A. 2004. An evolving view of duplex vision: Separate but interacting cortical pathways for perception and action. Current Opinion in Neurobiology 14, 203-211.

HisAKATA, R., AND MURAKAmi, I. 2009. Illusory position shift induced by plaid motion. Vision Research 49, 2902-2910.

Hommel, B., Müsseler, J., Aschersleben, G., AND Prinz, W. 2001. The theory of event coding (tec): A framework for perception and action planning. Behavioral Brain Sciences. 24, 849-937.

Kelly, D. H. 1979. Motion and vision. II. stabilized spatiotemporal threshold surface. Journal of the Optical Society of America 69(10), 1340-1349.

Kerzel, D., And Gegenfurtner, K. R. 2005. Motion-induced illusory displacement reexamined: Differences between perception and action? Experimental Brain Research 162, 191-201.

Kerzel, D., Gaugh, A., And Ulmann, B. 2008. Local motion inside an object affects pointing less than smooth pursuit. Experimental Brain Research 191, 187-195.

Khurana, B., And Nijhawan, R. 2010. Space and Time in Perception and Action. Cambridge University Press.

Mather, G., And Pavan, A. 2009. Motion-induced position shifts occur after motion integration. Vision Research 49, 27412746.

MiALL, R., AND JACKSON, J. 2006. Adaptation to visual feedback delays in manual tracking: evidence against the smith predictor model of human visually guided action. Experimental Brain Research 172, 77-84.

Milner, A. D., AND Goodale, M. A. 1995. The visual brain in action. Oxford University Press. 
MÜsseler, J., AND ASCHERsleben, G. 1998. Localizing the first position of a moving stimulus: The fröhlich effect and an attention-shifting explanation. Perception \& Psychophysics 60, 683-695.

NAKAYAMA, K. 1985. Biological image motion processing: A review. Vision Research 25(5), 625-660.

NiJHAWAN, R. 1994. Motion extrapolation in catching. Nature $370,256-257$.

NishidA, S., AND JOHNSTON, A. 1999. Influence of motion signals on the perceived position of spatial pattern. Nature 397(6720), 610-612.

PRINZ, W. 1997. Perception and action planning. European Journal of Cognitive Psychology 9, 129-154.

RAMACHANDRAN, V., AND ANSTIS, S. 1990. Illusory displacement of equiluminous kinetic edges. Perception 19, 611-616.

Rider, A., McOwan, P., And Johnston, A. 2009. Motioninduced position shifts in global dynamic gabor arrays. Journal of Vision 9(13):8, 1-8.

SmeEts, J., AND BREnNer, E. 2001. Action beyond our grasp. Trends in Cognitive Sciences 5, 287.

SNOWden, R. J. 1998. Shifts in perceived position following adaptation to visual motion. Current Biology 8, 1343-1345.

Thornton, I. M. 2002. The onset repulsion effect. Spatial Vision $15,219-243$.

Tsui, S., KhuU, S., And Hayes, A. 2007. The perceived position shift of a pattern that contains internal motion is accompanied by a change in the patterns apparent size and shape. Vision Research 47(3), 402-410.

Welch, R. B. 1978. Perceptual modification: Adapting to altered sensory environments. Academic Press.

Whitney, D. V., And Cavanagh, P. 2000. Motion distorts visual space: shifting the perceived position of remote stationary objects. Nature Neuroscience 3, 954-959.

Whitney, D., AND CAVANAGH, P. 2002. Surrounding motion affects the perceived locations of moving stimuli. Visual Cognition $9,139-152$.

Whitney, D., Goltz, H. C., Thomas, C. G., Gati, J. S., Menon, R. S., And Goodale, M. A. 2003. Flexible retinotopy: Motion-dependent position coding in the visual cortex. Science 302, 878-881.

Whitney, D. 2002. The influence of visual motion on perceived position. Trends in Cognitive Sciences 6, 211-216.

YAmagishi, N., Anderson, S. J., AND Ashida, H. 2001. Evidence for dissociation between the perceptual and visuomotor systems in humans. Proceedings of the Royal Society B: Biological Sciences 268, 973-977. 
